\title{
Interventions aimed at healthcare professionals to increase the number of organ donors: a systematic review
}

\author{
Marloes Witjes ${ }^{1,2}$, Nichon E. Jansen², Johannes G. van der Hoeven ${ }^{1}$ and Wilson F. Abdo ${ }^{1 *}$
}

\begin{abstract}
Background: The last decade, there have been many initiatives worldwide to increase the number of organ donors. However, it is not clear which initiatives are most effective. The aim of this study is to provide an overview of interventions aimed at healthcare professionals in order to increase the number of organ donors.

Methods: We systematically searched PubMed, EMBASE, CINAHL, PsycINFO, and the Cochrane Library for English language studies published until April 24, 2019. We included studies describing interventions in hospitals aimed at healthcare professionals who are involved in the identification, referral, and care of a family of potential organ donors. After the title abstract and full-text selection, two reviewers independently assessed each study's quality and extracted data.
\end{abstract}

Results: From the 18,854 records initially extracted from five databases, we included 22 studies in our review. Of these 22 studies, 14 showed statistically significant effects on identification rate, family consent rate, and/or donation rate. Interventions that positively influenced one or more of these outcomes were training of emergency personnel in organ donation, an electronic support system to identify and/or refer potential donors, a collaborative care pathway, donation request by a trained professional, and additional family support in the ICU by a trained nurse. The methodological quality of the studies was relatively low, mainly because of the study designs.

Conclusions: Although there is paucity of data, collaborative care pathways, training of healthcare professionals and additional support for relatives of potential donors seem to be promising interventions to increase the number of organ donors.

Trial registration: PROSPERO, CRD42018068185

Keywords: Organ donation, Organ donor rate, Intensive care, Family guidance, Donor identification, Donor referral, Consent rate

\section{Background}

The large gap between organ donor availability and organ demand is a major healthcare issue worldwide. In 2017, the Netherlands had 15.2 actual deceased organ donors per million population (PMP), while at the end of 2017, there were still 1138 patients awaiting a transplant and 140 patients who died while on the waiting list [1]. The UK had 22.5 deceased organ donors PMP, 6739

\footnotetext{
* Correspondence: f.abdo@radboudumc.nl

'Department of Intensive Care Medicine, Radboud Institute for Health Sciences, Radboud University Medical Center, P.O. Box 9101, Internal post 710, 6500 HB Nijmegen, The Netherlands

Full list of author information is available at the end of the article
}

patients awaiting a transplant and 436 patients died while on the waiting list. For the USA, 31.7 deceased organ donors PMP, 77,115 patients were on a transplantation waiting list, and 6021 patients died while on the waiting list [1]. To amend the large organ donor shortage and increase organ donation rates, many initiatives have been suggested. These initiatives range from changing the legal consent system (opt-in versus opt-out) [2], large-scale public campaigns to raise awareness [3], to interventions aimed at increasing the organ donation pool through expanding medical criteria [4]. Due to the continuing shortage, new interventions are proposed frequently.

(c) The Author(s). 2019 Open Access This article is distributed under the terms of the Creative Commons Attribution 4.0 International License (http://creativecommons.org/licenses/by/4.0/), which permits unrestricted use, distribution, and 
Many studies have also been published on improving the donation process in the hospital [5]. The donation process starts with the identification of a potential organ donor. Subsequently, the potential organ donor must be referred to the intensive care unit (ICU), if not already admitted, and the organ procurement organization (OPO). In addition, irrespective of the legal consent system, consent must be obtained either by the donor himor herself via the Donor Registry and/or by family members. Healthcare professionals play an important role in the donation process as they are directly involved and responsible for identifying and referring potential organ donors and obtaining consent. Several studies have shown that potential organ donors are not always recognized, especially when patients die outside the ICU [6-8]. For example, a study from the Netherlands described that the number of unrecognized organ donors outside the ICU was $11-34 \%$ of the known organ donor pool $[6,9]$.

The objective of this study was to pinpoint effective interventions that were aimed at healthcare professionals and had the goal of increasing the number of organ donors.

\section{Methods}

A systematic review of the literature was performed. The criteria for article inclusion and data analysis were prespecified. The initial protocol has been registered in PROSPERO, the international prospective register of systematic reviews with registration number CRD42018068185 [10].

\section{Data sources and searches}

PubMed (including MEDLINE), EMBASE, CINAHL, PsycINFO, and Cochrane Library were searched until April 24, 2019, restricted to English language publications. The search strategy included the following concepts: post-mortem organ donation, healthcare professionals, and interventions in hospitals. The complete search strategy for each database is presented in Additional file 1. The author's personal files and references of included studies were also searched to identify additional relevant articles (snowballing).

\section{Selection criteria and process}

Titles and abstracts retrieved from the search strategy were independently screened by two authors (MW and $\mathrm{NJ})$, to identify studies that potentially fulfilled inclusion criteria. Full-text articles were screened by the same two authors. Disagreement on inclusion was resolved by discussion. Studies were included when they met all the following inclusion criteria:

1. The healthcare intervention was aimed at healthcare professionals who were involved in the identification, referral, and support of (relatives of) potential organ donors.
2. Study design was experimental, quasi-experimental, or observational, such as randomized controlled trials, (un) controlled before-after studies, and (non-) controlled cohort studies.

3. Study had at least one quantitative outcome measure.

4. English language full-text article is available.

Studies were excluded when the interventions aimed to increase the potential donor pool or improve logistics outside the hospital, e.g., implementation of a donation after circulatory death (DCD) protocol, implementation of a donation program with transplant coordinators and regional retrieval teams, expanding the donor pool (older donors, DCD donors, non-ventilated donors), education of the population, ways to recover organ function, legislative measures, and improved allocation algorithms. In addition, we excluded donor programs consisting of more than two interventions. Although a bundle might be interesting as an approach, the effect per individual intervention cannot be distinguished. Also, a bundle would mean all components of the bundle have to be implemented to obtain the effect making it more difficult to implement in daily practice.

\section{Data extraction and quality assessment}

One researcher (MW) extracted the data from the included studies, using a standardized form (see Additional file 2). The extracted data were checked by a second researcher $(\mathrm{NJ})$. The extracted data included the study design, objective and methods, setting, population and sample size, intervention, outcomes and results, conclusion, and article comments from the reviewers.

The quality of the included articles was assessed using the suggested risk of bias criteria for EPOC (Effective Practice and Organisation of Care) reviews from the Cochrane Handbook [11]. The criteria for studies with a separate control group (randomized trials, non-randomized trials, and controlled before-after studies) were different from the criteria for studies without a control group (uncontrolled beforeafter studies, cohort studies). Quality criteria were independently assessed by two authors (MW and NJ). Discrepancies were resolved by discussion between these two authors.

\section{Data synthesis and analysis}

We tabulated study characteristics and outcomes such as study design, intervention, number of participants, outcomes, and significance level. The interventions were described in more detail and classified in one or more of the following categories: (1) identification and referral of the potential organ donor, (2) education of the healthcare professionals, and (3) extra support of the relatives to help them make a well-considered decision on donation. 


\section{Results}

Our search identified 18,854 records, of which 5515 duplicate records were removed (Fig. 1). In total, 13,339 records were screened for title abstract. After excluding 13,295 records, 44 full-text articles were assessed for eligibility. The final set of articles consisted of 22 full-text articles. No new articles were identified through snowballing.

\section{Characteristics of included studies}

Table 1 summarizes study characteristics and outcomes of the 22 studies. Fourteen uncontrolled before-after studies (UBAs), four cohort studies, two randomized controlled trials (RCTs), one controlled before-after study (CBA), and one non-randomized controlled trial (NRCT) were analyzed. The interventions were aimed at healthcare professionals: physicians, nurses (specialized in donation), requestors, personnel from the procurement centers, social workers, chaplains, administrators, and psychologists. Ten studies were single-center studies, and 12 studies were performed across multiple hospitals ranging from 3 to 220 hospitals. The study population consisted of relatives of ICU patients, potential/eligible donors, emergency department (ED) deaths, declared brain deaths, donation requests, and patients meeting trigger criteria. The sample size per study ranged from 11 to 1101 in the intervention group and from 3 to 1563 in the control group. The majority of the cases were DBD and most studies included donation after brain death as well as DCD, depending on whether the country had a DCD protocol.

Various outcomes were reported: donor identification, donor referral (from the ED), family approach rate, consent rate, donation rate, and organs recovered. Most interventions were aimed at increasing the referral rate (from the ED), consent rate or donation rate (which is based on the referral and consent rate). Significant differences were seen in all of these three outcomes. In eight studies, the significance level was not reported. If possible, we calculated the $p$ value with the data that were available (Tables 1 and 2).

\section{Methodological quality}

The results from the quality assessment are shown in Additional file 3. Overall, the quality of the studies was relatively low, mainly because of the study designs that

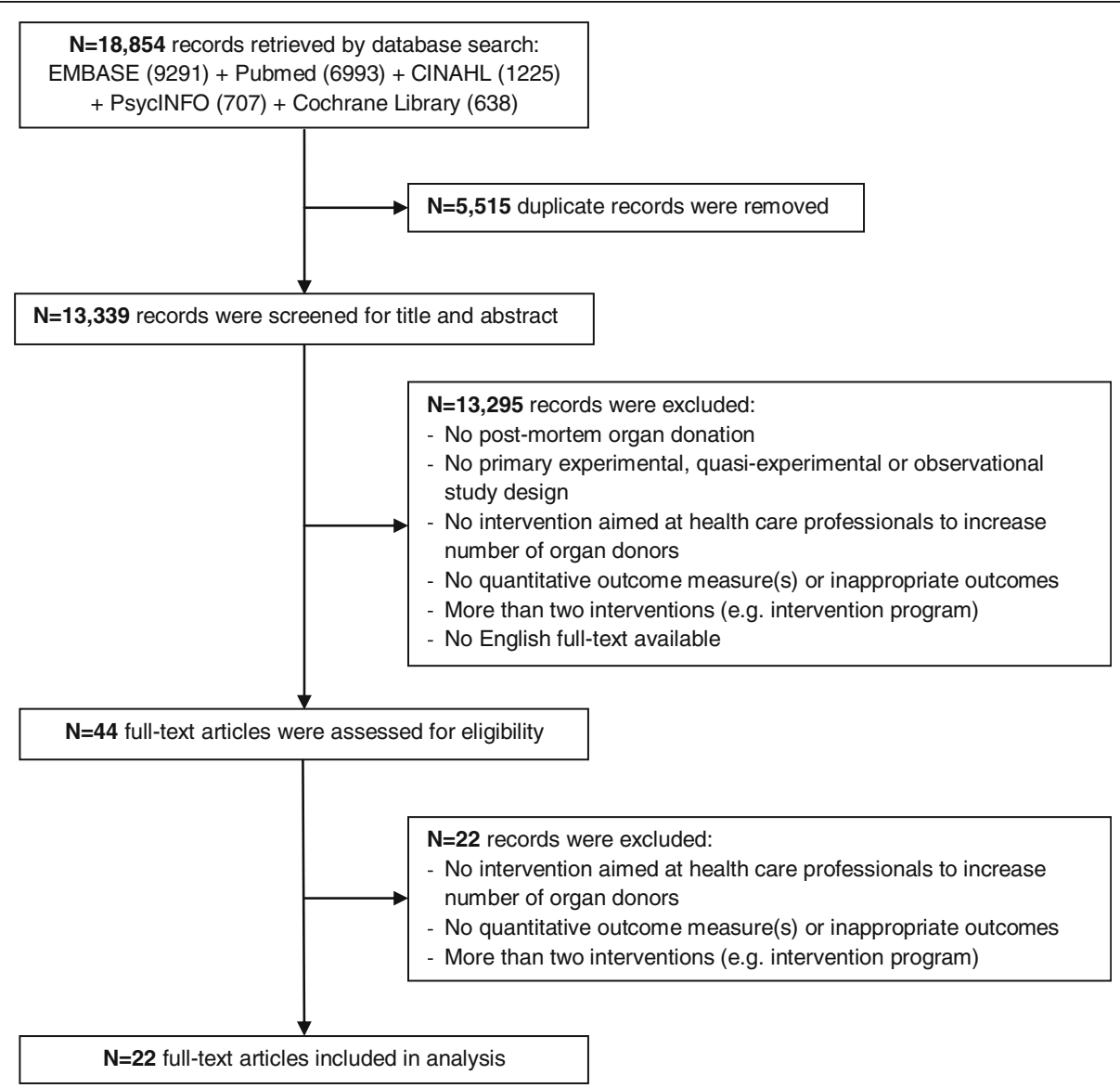

Fig. 1 Flow chart showing the inclusion of articles 


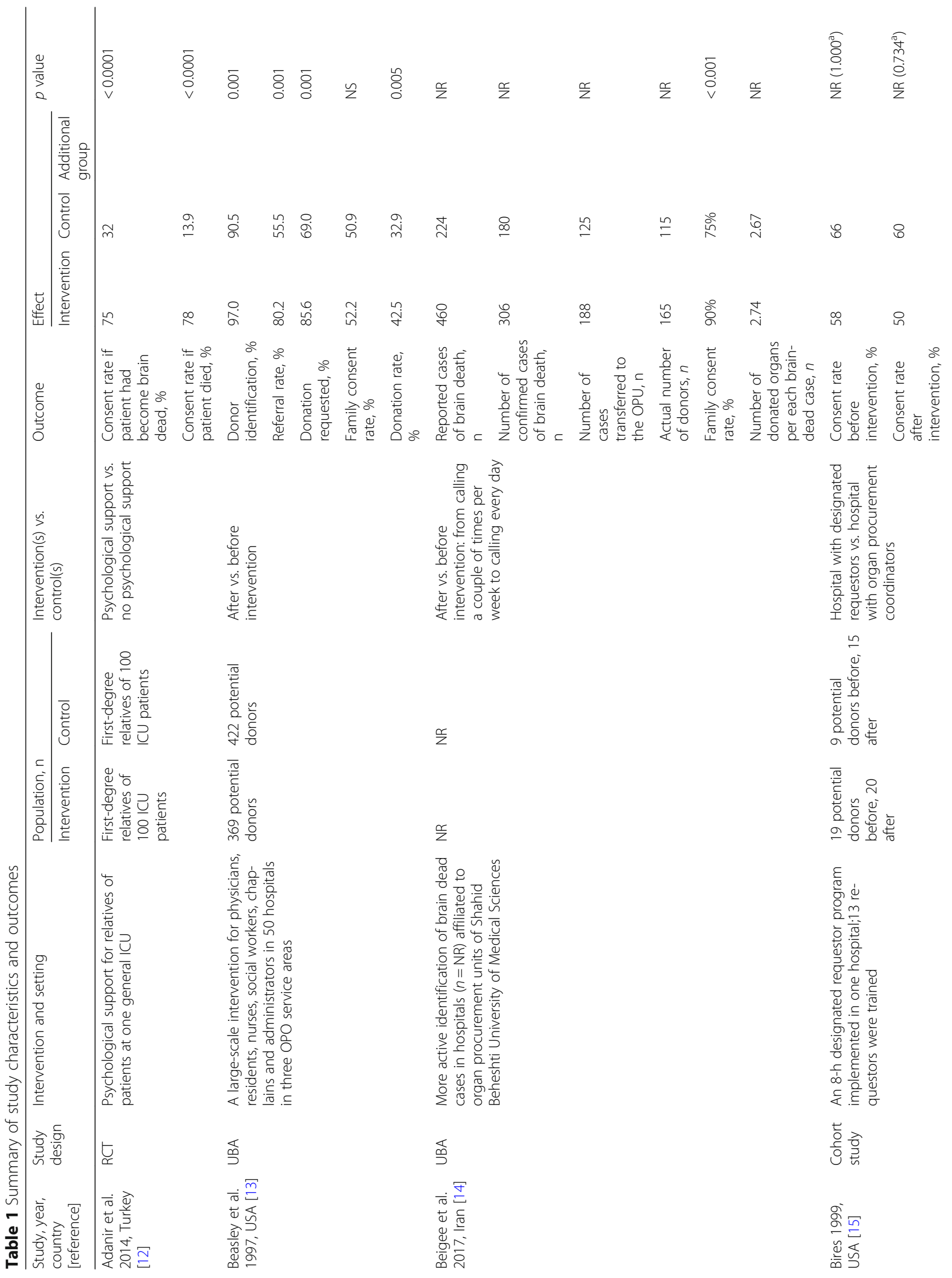


Witjes et al. Critical Care $\quad$ (2019) 23:227

Page 5 of 19

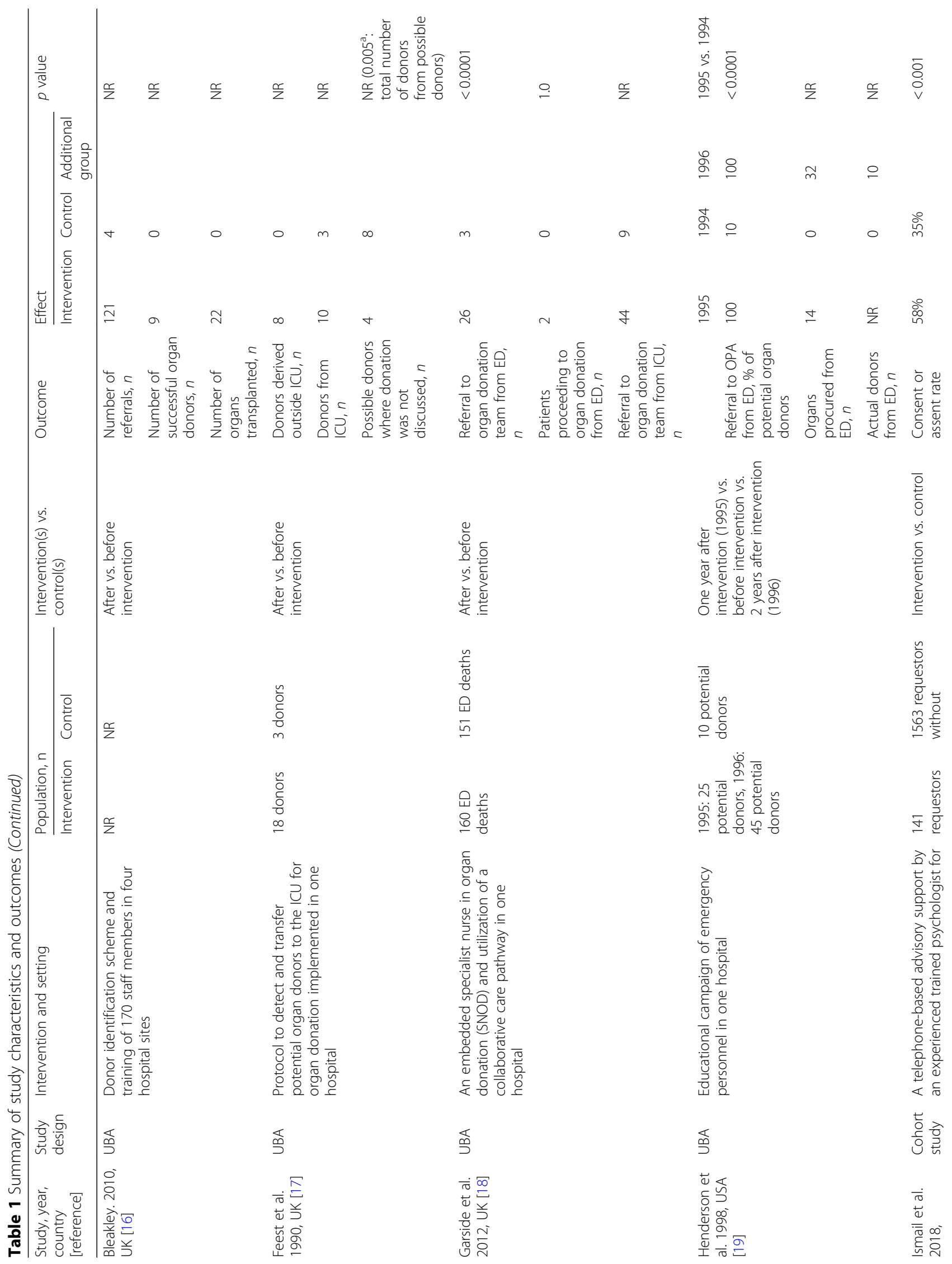




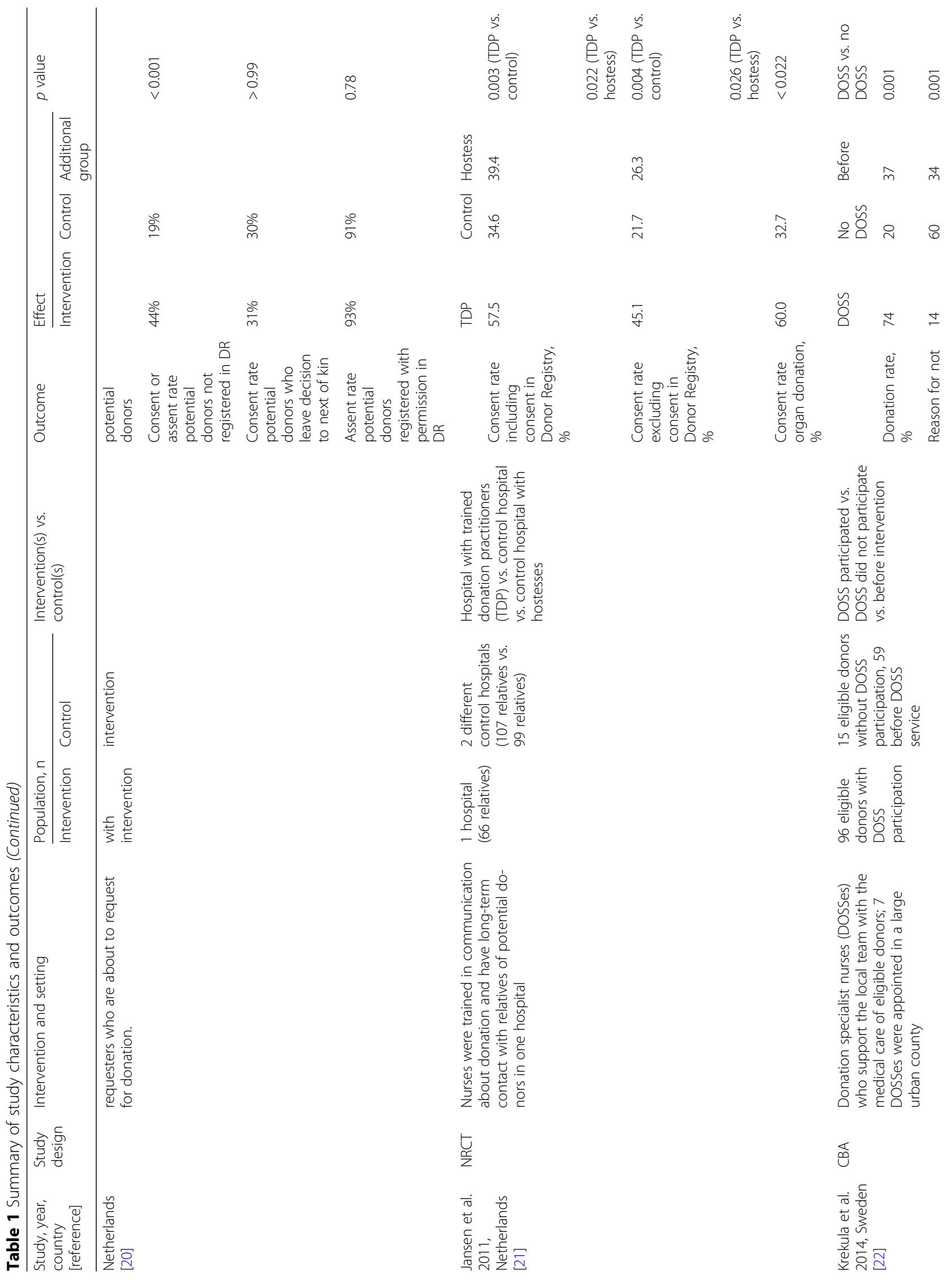




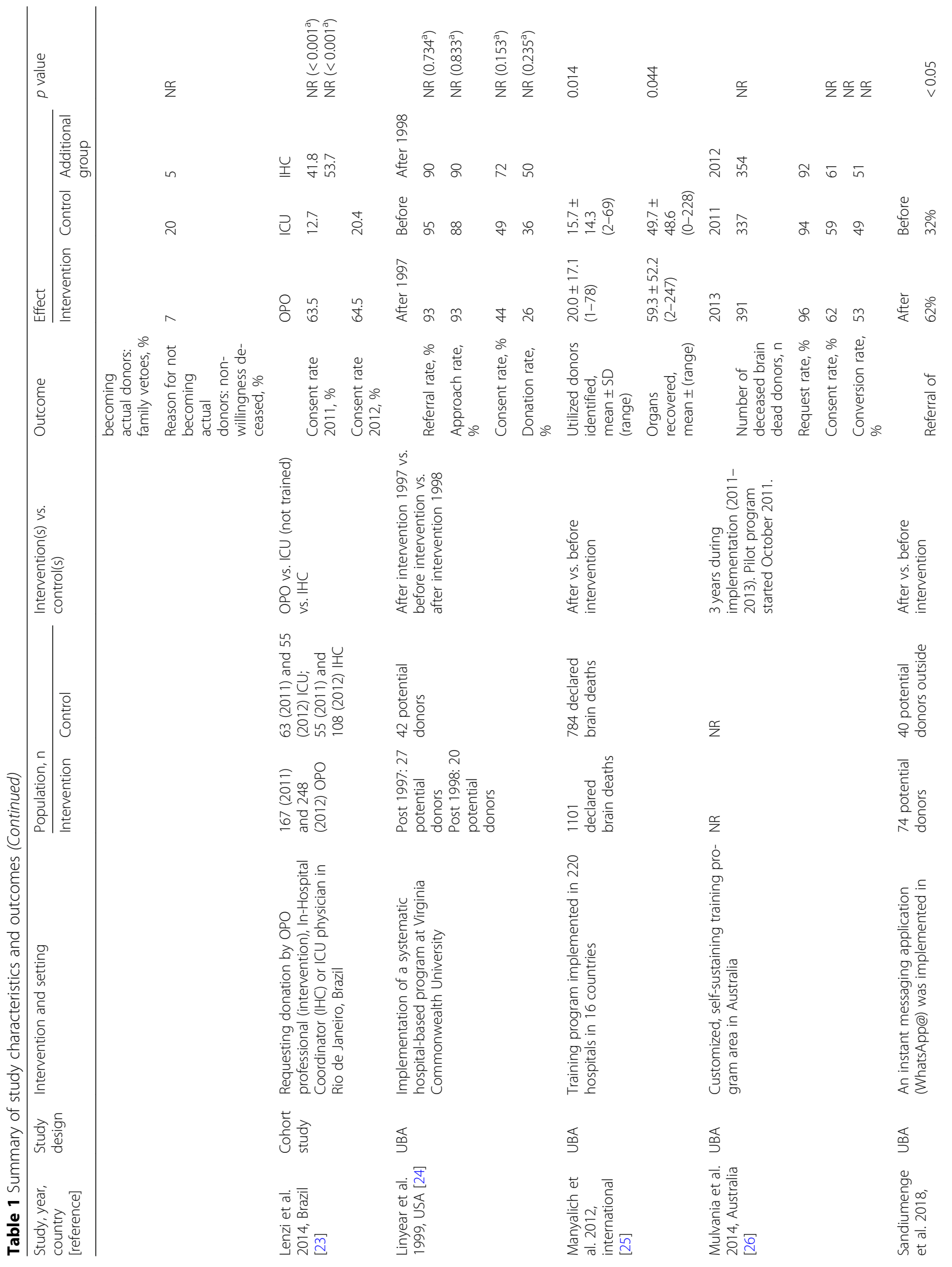




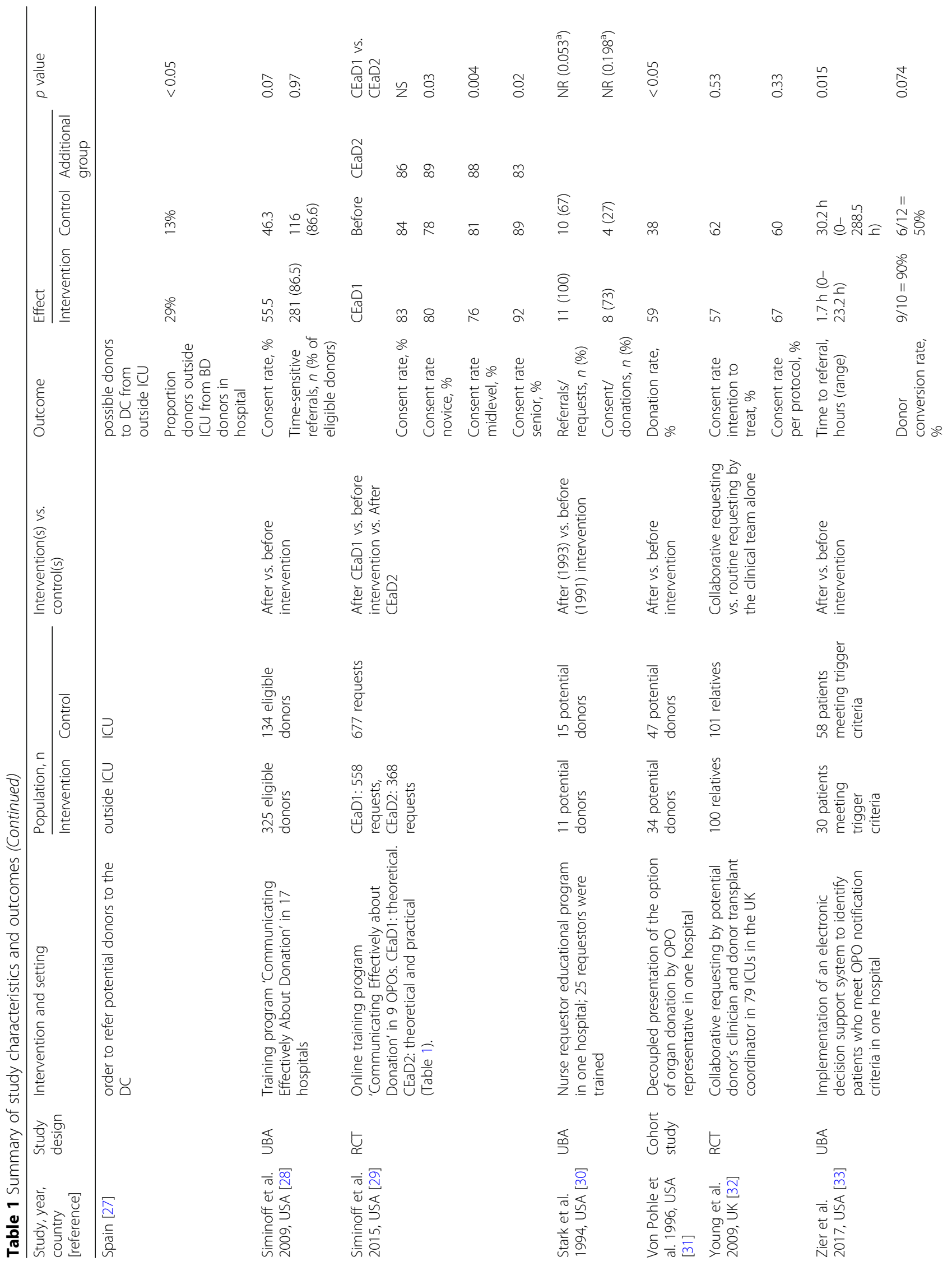


Witjes et al. Critical Care $\quad$ (2019) 23:227

Page 9 of 19

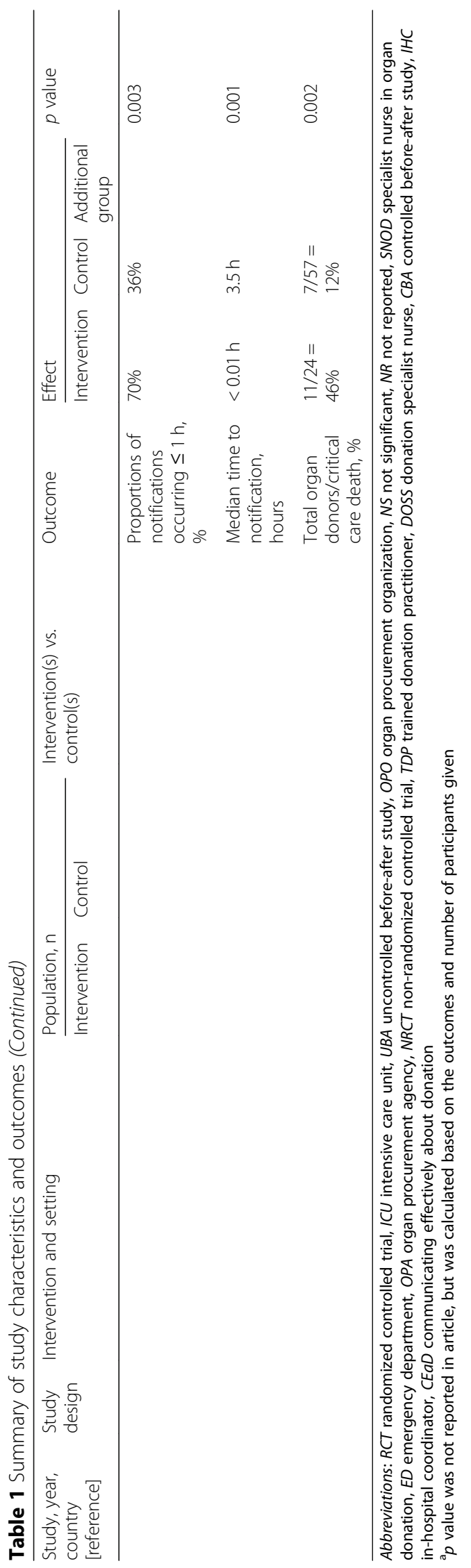




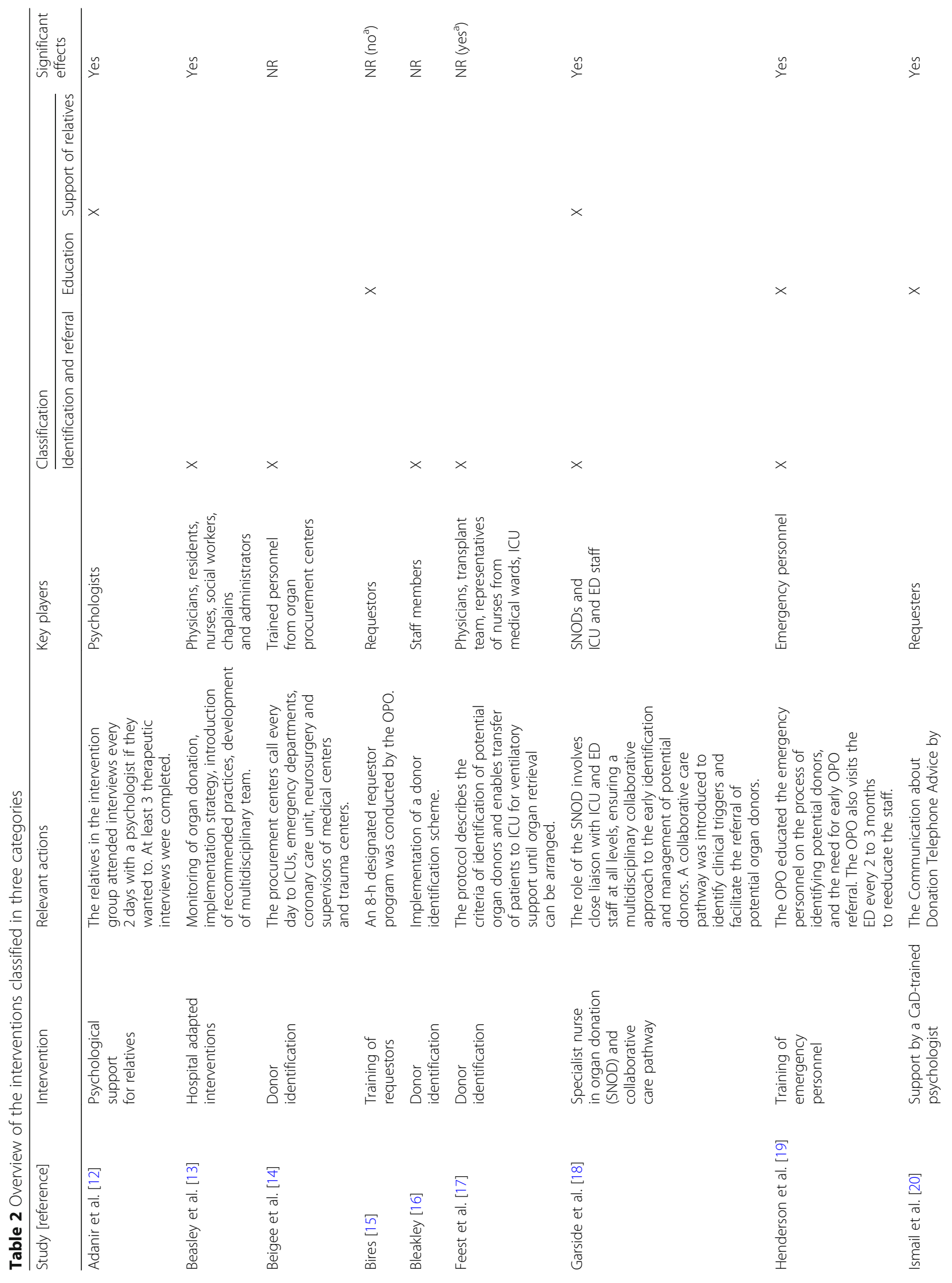




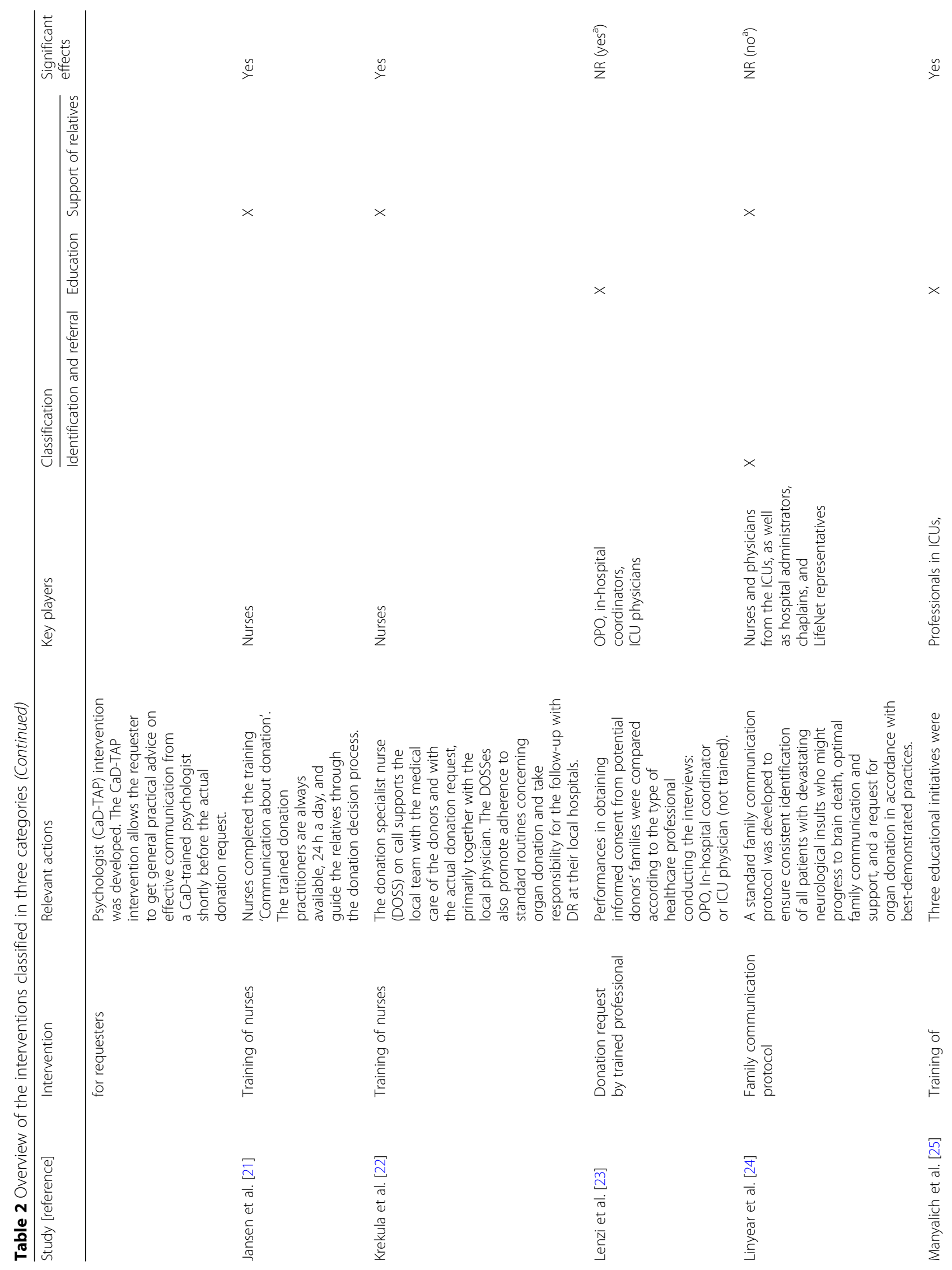




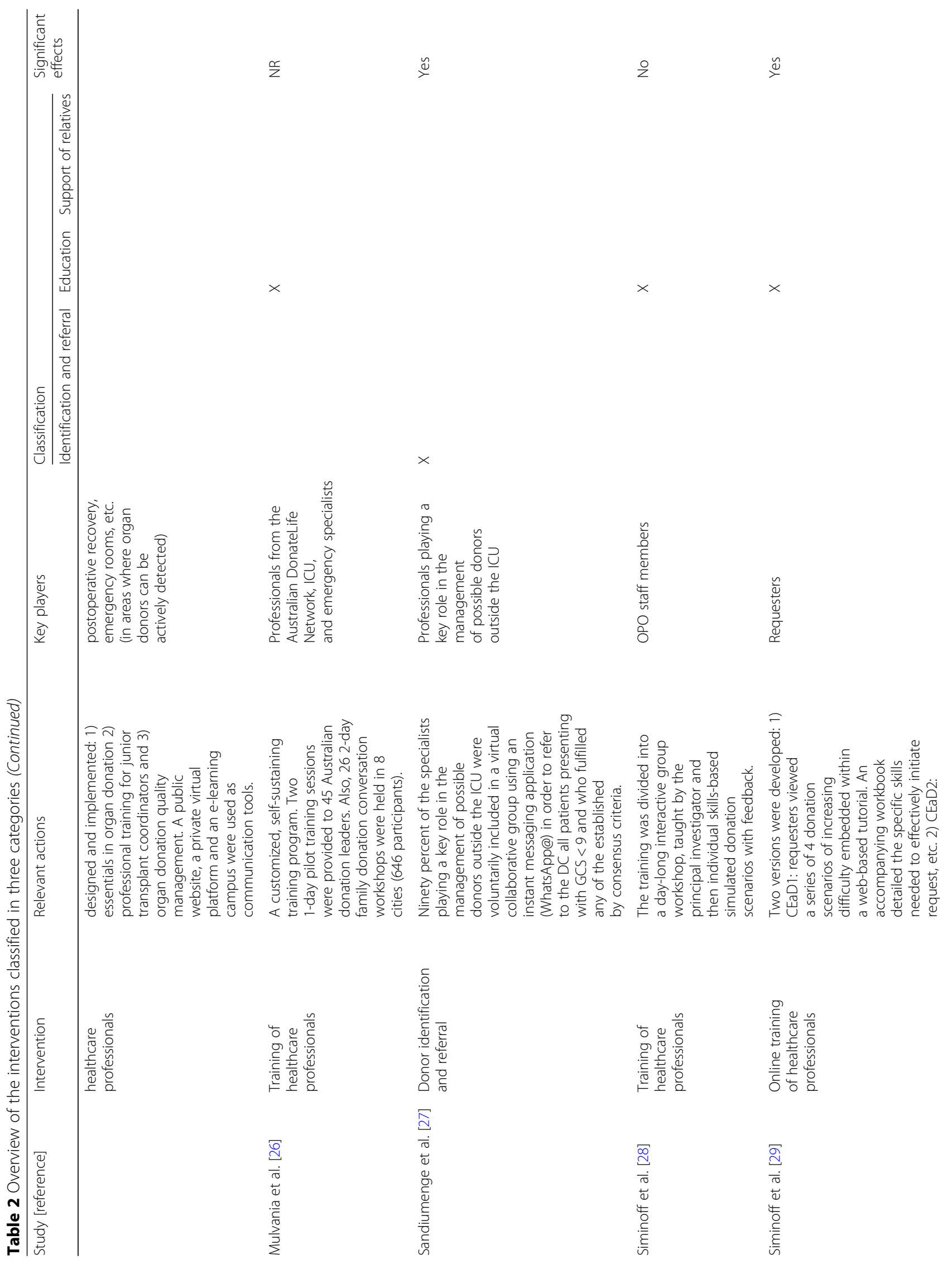




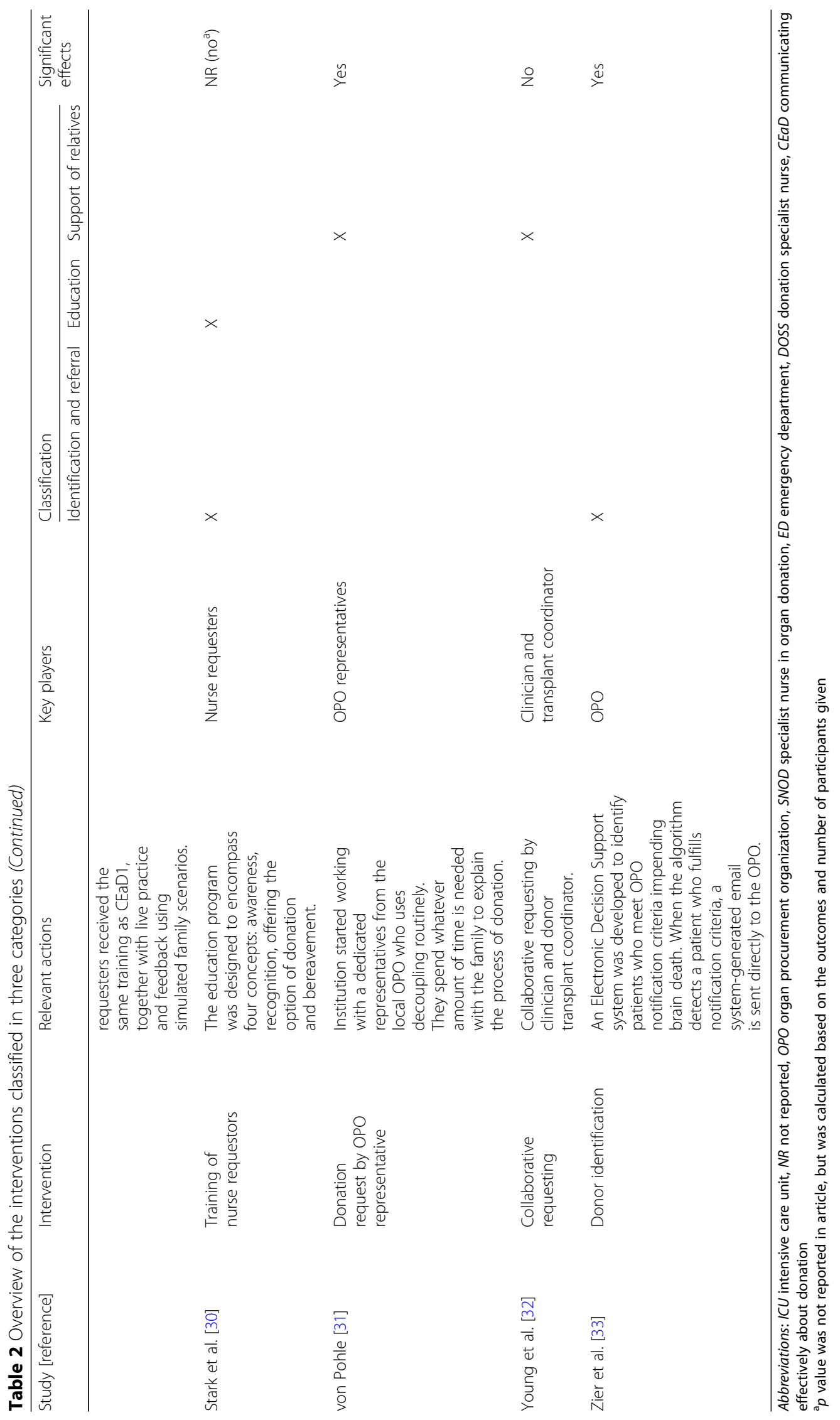




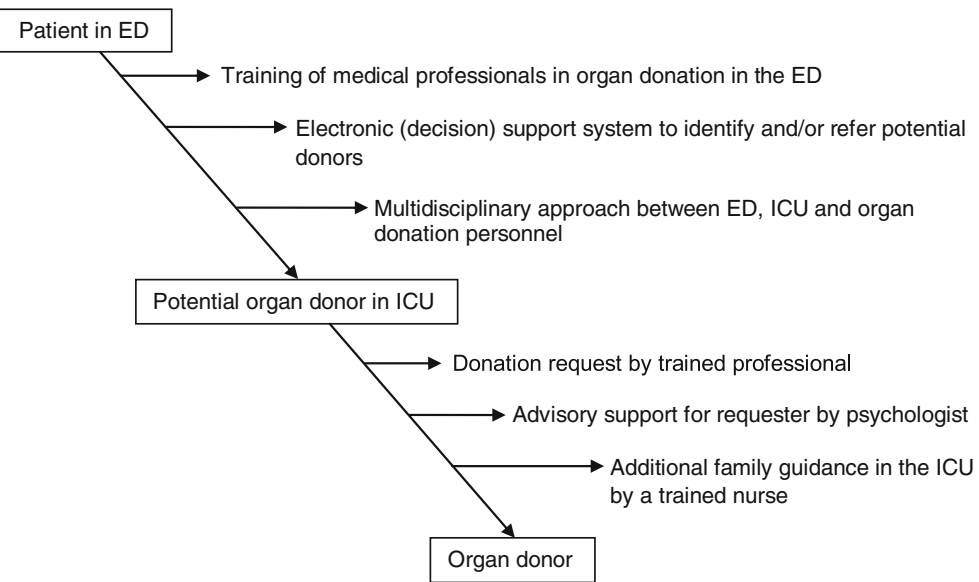

Fig. 2 Areas with beneficial interventions focused on healthcare professionals. Abbreviations: ED, emergency department, ICU intensive care unit

were used. Seventeen studies did not use a control group, which makes the criterion "intervention independent of other changes" for these studies at high risk. Two RCTs were performed [12, 32]. One of these studies did not use a correct randomization method. They divided the groups by even and odd numbered beds [12]. In addition, most studies lacked adequate power or had selection bias leading to a high-risk score on the criterion "other risks of bias."

The studies found in this systematic review were not suitable for a meta-analysis due to heterogeneity in interventions and outcome, the different definitions used for "potential donor" [34, 35], and different (legal) systems used in every country.

\section{Effects of the interventions}

Table 2 provides an overview of the interventions identified in the 22 articles, classified in 3 categories of interventions: identification and referral, education, and extra support of the relatives. Some interventions could be classified in more than one category.

\section{Identification and referral}

Ten studies focused on the identification and referral of potential organ donors [13, 14, 16-19, 24, 27, 30,33]. Six out of ten studies focused on donor identification and referral [13, 14, 16, 17, 27, 33]. Two of these six studies showed statistically significant higher identification rates $[13,33]$ after the intervention. In the study by Beasley et al. [13], a multidisciplinary strategy was introduced in 50 hospitals which increased the donor identification from 90.5 to $97.0 \%(p=0.001)$ and the donation rate from 32.9 to $42.5 \%(p=0.005)$. In the study by Zier et al. [33], the donation rate increased from 12 to $46 \%$ by implementing an electronic decision support system to identify potential organ donors $(p=0.002)$. The study by Sandiumenge et al. [27] used technology to refer potential organ donors to the donation coordinator (DC). Ninety percent of the specialists playing key role in the management of possible donors outside the ICU were included in a WhatsApp group in order to refer to the DC. After the intervention, $62 \%$ of the possible donors outside the ICU were referred to the DC compared to $32 \%$ before the intervention $(p<0.05)$. These referred donors after the intervention had a mean age of 72 years, and the main cause of death was hemorrhagic stroke (59\%) followed by ischemic stroke (33\%). Three of the six studies which focused solely on donor identification showed an increase in the number of referrals; however, it was not reported whether this was statistically significant $[14,16,17]$. The studies of Bleakly et al. [16] and Feest et al. [17] focused on donor identification by implementing an identification scheme describing the criteria of identification for personnel in the ED. In the study by Beigee et al. [14], procurement centers called the hospital departments every day to check if there were any potential donors. This resulted in an increase in the number of brain death cases that were transferred to the OPU from 125 to 188 . The mean age of these 188 donors was 45 years. In most cases, the cause of brain death was a cerebrovascular accident (47\%).

Four out of ten studies, focusing on identification and referral, also focused on education $[19,30]$ or support of relatives $[18,24]$. The study by Henderson et al. [19] showed that a training for emergency personnel on the process of identifying potential donors significantly increased the referral rate from 10 to $100 \%$ and the number of actual donors from 0 to 10 ( $p=$ not reported). A nurse requester education program led to an increased donation rate, however not statistically significant [30]. In the study by Garside et al. [18], an embedded specialist nurse in organ donation (SNOD) and a collaborative pathway was introduced to identify clinical triggers and facilitate the referral of potential organ donors. This led 
to an increase in referral from the ED from 3 to 26 ( $p<$ $0.0001)$. It did, however, not lead to a significant increase in organ donors from the ED (0 to 2). The family communication protocol that was introduced in the study by Linyear et al. [24], did not lead to an increased referral rate, but showed a non-significant increase in donation rate from 36 to $50 \%$ in 2 years after the introduction $(p=0.235)$.

\section{Education of healthcare professionals}

In total, nine studies focused on education of healthcare professionals $[15,19,20,23,25,26,28-30]$. A cohort study by Ismail et al. [20] showed that advisory support by a trained psychologist for requesters who are about to request for donation increased the family consent rate from 35 to $58 \%(<0.001)$. A retrospective cohort study by Lenzi et al. [23] showed that when family conversations were done by an OPO representative or in-hospital coordinator, this led to significantly higher consent rates than when this was done by non-trained ICU physicians (respectively $64.5 \%, 53.7 \%$, and $20.4 \%$ ). In the study by Manyalich et al. [25], an advanced training program was implemented in 220 hospitals in 16 countries, which was adapted to the country's needs. This training program consisted of three educational initiatives: essentials in organ donation, professional training for junior transplant coordinators, and organ donation quality management. Online communication tools were used to implement these initiatives. The results of this study showed an increase in the mean number of utilized donors identified from 15.7 to $20.0(p=0.014)$ and the mean number of organs recovered from 49.7 to 59.3 $(p=0.044)$. Siminoff et al. [28] designed a training program for OPO staff members consisting of a day-long interactive workshop and individual skills-based simulated donation scenarios with feedback. The training led to an increase in consent rate from $46.3 \%$ to $55.5 \%$ ( $p=$ 0.07 ). This study was followed by another study by Siminoff et al. [29], where two online versions of the training program were developed. Overall, this did not lead to an increase in consent rate ( $84 \%$ before intervention, $83 \%$ after intervention).

\section{Additional support of relatives}

Seven studies focused on additional support of relatives [12, 18, 21, 22, 24, 31, 32]. Six out of seven studies showed a statistically significant increase in the main outcome measure after the intervention. In the study by Adanir et al. [12], psychologists performed therapeutic sessions with the relatives of ICU patients. Although the relatives were not relatives of potential organ donors, the percentage of people that would consent to donation, if they had to decide, was higher in the intervention group (75\%) than the control group (32\%) with $p<$
0.0001. In the study by Jansen et al. [21], nurses were trained who were available $24 \mathrm{~h}$ a day to guide the relatives of potential donors. A significantly higher consent rate was seen in the intervention hospital with the trained nurses (57.6\%), than in the two control hospitals (34.6\% and $39.4 \%)$. The mean age of the potential donors in the intervention group was 63 years. The study by Krekula et al. [22] also showed an increase in donation rate when working with trained nurses (called "donation specialist nurse" (DOSS)), first in a DOSS local project and later in a DOSS county-based service (Table 1 shows the outcomes of the DOSS service). In the ACRE trial [32], it was shown that collaborative requesting by a clinician and donor transplant coordinator did not increase the consent rate when compared to requesting by the clinical team alone ( $57 \%$ vs. $62 \%, p=0.53$ ).

In Fig. 2, the beneficial interventions are summarized and visually displayed per area.

\section{Discussion}

This systematic review provides an overview of interventions aimed at healthcare professionals in order to increase the number of organ donors. Interventions, with statistically significant effects, were found in all three categories: identification and referral, education, and extra support of relatives, where some interventions focused on two categories. However, the results we found were based on studies with a relatively low methodological quality. Also, many of the included studies were with insufficient power. The lack of high-quality studies seems typical for the research area and for research that is being developed and implemented in practice.

We found that implementation of a collaborative approach between the ICU and other departments, such as the department of neurology, ED, and neurosurgery ("collaborative care pathway") to identify triggers, facilitates identification and referral of potential organ donors $[16,18,24]$. Implementing such a collaborative care pathway creates the possibility to make organ donation part of end-of-life care, also outside the ICU. Recently, more literature, including studies that were not included in this systematic review, has been published on this topic [5, 6, 36-39]. A recent paper by Martinez-Soba et al. described their experience with an Intensive Care to facilitate Organ Donation (ICOD) protocol [38]. They retrospectively reviewed patients with a devastating brain injury whose families were approached to discuss the possibility of ICOD. This also included patients in which the decision was made not to intubate $(50 \%$ of the cases). The results showed that ICOD was well accepted by families and ICOD contributed to 33\% of the actual donors. Another recent paper by Witjes et al. describes their experience with the implementation of a multidisciplinary approach in the ED. They describe that organ 
donors from the ED with a fatal brain injury are an important portion $(29 \%)$ of the total pool of organ donors. Although such an intervention is not straightforward to implement due to its multidisciplinary approach, it could lead to improved donation awareness and better donor identification in the ED.

In some studies, a large number of referrals did not lead to effected organ donors. For instance, in the study by Bleakley et al. [16], it was shown that an increase in referrals to the on-call donor transplant coordinator from 4 to 121 referrals led to an increase in organ donors from 0 to 9 . This means that per organ donor, 13 referrals to the OPO were needed (donation rate of $7.4 \%)$. A difficulty in analyzing such data is that not each referred patient was actually a potential organ donor and that there are international differences between the definition of a potential organ donor [34, 35].

With regard to the organ donation request, most studies showed that the professional requesting donation should be trained, although not all studies showed a significant effect, mostly due to low sample size $[15,28$, 30]. The person who is requesting for donation also differs per country. For example, in the USA, the OPO (who is also involved in the transplant side) is requesting for donation, and in the UK, it is the SNOD who is requesting for donation (and is not involved in the transplant side). In many countries, the requester is part of the treating team and is mostly an ICU physician.

Much research has been published on factors in the donation process that influence consent rates [40-48]. This research also showed that the skills of the requester influence the consent rate, just as the information discussed during the request, understanding of brain death, timing of the request, setting in which request is made, characteristics of the requester, the family 's satisfaction level with the medical attention, et cetera. All this information was used to develop various educational programs for healthcare professionals involved in donation practices, some of them shown in our review. In addition, in a large nationwide study including 1322 organ donation requests, it was recently shown that when the requesting physician contacted the OPO before the organ donation request and discussed the case, this led to a higher consent rate [48]. This was presumably because the contact between requesting physician led to more clarity in the conversations with the family as the requesting physician could provide more specific information regarding (suitability for) donation and approximation of the time span of logistics surrounding organ donation.

With regard to family guidance, we found that additional support of relatives by healthcare professionals increased the consent and donation rates. The healthcare professionals were mostly nurses who were trained in organ donation in order to support the relatives of potential organ donors in their decision-making process.
On the other hand, collaborative requesting by clinician and donor transplant coordinator did not increase consent rate [32].

\section{Limitations}

Our systematic review has several limitations. First, the studies included in our review are mostly uncontrolled before-after studies which tend to overestimate the effect. On the other hand, these study designs are more feasible in practice than randomized controlled trials, since blinding and concealment of allocation are often not achievable in this area. Second, we did not include articles that reported effects of combined interventions as the effect per individual intervention could not be distinguished, making it harder to implement such an intervention in the daily practice. An earlier review [49] (with articles until 2010) on interventions aimed at healthcare professionals, did evaluate these donor programs with combined interventions and found that the evidence of the 15 included articles was weak due to methodological flaws, as a vague definition of the intervention, lack of explanations on the study design, and unjustified sample size. Third, we only included full-text articles available in English, which may have increased the risk of publication bias. Fourth, many of the included articles dated back to $>20$ years ago. This could make their data less applicable to the current practice.

The paucity of data in peer-reviewed journals does not mean that there is no evidence for successful initiatives to increase the number of organ donors. Although not the focus of our review, there have been successful donor programs (with combined best practices) that have improved and sustained organ donation [50,51]. The focus on potential organ donors outside the ICU, e.g., ED, could be an area where a collaborative effort between the ICUs and ED can increase the number of organ donors and more data is needed from successful collaborative efforts [6, 37-39]. Besides scientific evidence, other (policy) documents exist on interventions that could increase the number of potential organ donors [52]. However, much of these data are not published in peer-reviewed journals, which makes them more difficult to assess and compare to scientific standards. We would like to make a call to action to research, audit, and evaluate initiatives to improve organ donation practices, and to publish these results in scientific papers.

\section{Recommendations and future research}

Based on our extensive literature search, the following recommendations can be made.

With regard to the identification and referral of potential organ donors in the ED, we recommend that hospitals develop a process that ensures that all potential organ donors are identified. Most hospitals will already have such a systematic approach for patients in the ICU. 
However, such a systematic approach is mostly lacking for potential organ donors outside the ICU, e.g., the emergency department. Successful approaches focusing on this area included a close collaboration between the organ procurement staff, the ICU and departments involved outside the ICU such as the emergency department, the department of neurology, neurosurgery and traumatology. In addition, educating medical professionals outside the ICU in organ donation is paramount in such an approach. Important questions in such a collaborative approach are "who should make the organ donation request?" "where should the organ donation request be made (ED or ICU)?" "what logistical arrangements are required (and should be arranged beforehand) to admit potential donors to the ICU?"

Concerning the consent rate, it is important that the professional who is requesting for donation should be trained in communicating organ donation. In addition, ICU nurses could play an important role in guiding the family during and after the consent process.

It is known that clear communication and information about the donation process are crucial for the family. The role that ICU nurses or other professionals could play in family guidance needs further research as the scientific evidence is limited. Future research could also focus on technology that could be used to (automatically) identify and refer potential organ donors Although randomized controlled trials are difficult to perform in this area, it is important that future research studying new interventions also include control groups. Ideally, a control group is compared to an intervention group in the same time period and prospectively measured.

\section{Conclusions}

In conclusion, this systematic review describes interventions that lead to higher numbers of organ donors. The main finding is that collaborative care pathways, in which donor identification criteria are identified, training of healthcare professionals (also in the ED) and additional focus on support of relatives of potential donors, could be promising interventions to increase the number of organ donors. The paucity of data in peer-reviewed journals asks for a call to action to publish the results of initiatives to improve organ donation.

\section{Additional files}

Additional file 1: Search strategy for each database. This additional file shows the search strategies that were used in the different databases. (DOC $42 \mathrm{~kb}$ )

Additional file 2: Data extraction form. This extraction form was used to extract the data from the included articles. (DOC $246 \mathrm{~kb}$ )

Additional file 3: Quality assessment of the included studies according to suggested risk of bias criteria for Effective Practice and Organisation of
Care (EPOC) reviews [11]. This additional file shows the quality assessment of the included studies. (DOCX $16 \mathrm{~kb}$ )

\section{Abbreviations}

CBA: Controlled before-after study; DC: Donation coordinator; DCD: Donation after circulatory death; DOSS: Donation specialist nurse; ED: Emergency department; EPOC: Effective Practice and Organisation of Care; ICOD: Intensive Care to facilitate Organ Donation; ICU: Intensive care unit; NRCT: Nonrandomized controlled trial; OPO: Organ procurement organization; PMP: Per million population; RCT: Randomized Controlled Trial; SNOD: Specialist nurse in organ donation; UBA: Uncontrolled before-after study

\section{Acknowledgements}

We gratefully thank Elmie Peters from Radboud University library for her help with building the search strategies in the five databases.

\section{Authors' contributions}

MW, NEJ, JGH, and WFA conceived the study design and authored the study protocol. MW developed and performed literature searches. MW and NEJ screened references, extracted the data, and performed the quality assessments. MW wrote the manuscript and all authors contributed with revisions and gave approval to the final version of the manuscript.

Funding

This study was not funded.

Availability of data and materials

The datasets used and/or analyzed during the current study are available from the corresponding author on reasonable request.

Ethics approval and consent to participate

Not applicable

Consent for publication

Not applicable

\section{Competing interests}

The authors declare that they have no competing interests.

\section{Author details}

${ }^{1}$ Department of Intensive Care Medicine, Radboud Institute for Health Sciences, Radboud University Medical Center, P.O. Box 9101, Internal post 710, 6500 HB Nijmegen, The Netherlands. '2Dutch Transplant Foundation, Leiden, The Netherlands.

Received: 6 February 2019 Accepted: 6 June 2019

Published online: 20 June 2019

\section{References}

1. Newsletter Transplant 2017 of Organización Nacional de Trasplantes. http:// www.ont.es/publicaciones/Documents/NewsleTTER\%202018\%20final\%20CE. pdf. Accessed 24 Jan 2019

2. Shepherd $\mathrm{L}, \mathrm{O}^{\prime}$ Carroll RE, Ferguson $\mathrm{E}$. An international comparison of deceased and living organ donation/transplant rates in opt-in and opt-out systems: a panel study. BMC Med. 2014;12:131

3. Feeley $\mathrm{TH}$, Kruegler J. Promoting organ donation through challenge campaigns. Prog Transplant. 2015;25(2):176-81.

4. Rusinova K, Cerny V, Kukal J, Pokorna E. Expanding the DCD donor pool: prediction of time to death after removal of life-sustaining treatments. Intensive Care Med. 2015;41(9):1719-20.

5. Dominguez-Gil B, Murphy P, Procaccio F. Ten changes that could improve organ donation in the intensive care unit. Intensive Care Med. 2016;42(2):264-7.

6. Witjes M, Kotsopoulos A, Herold IHF, Otterspoor L, Simons KS, van Vliet J, et al. The influence of end-of-life care on organ donor potential. Am J Transplant. 2017:17(7):1922-7.

7. Le Conte P, Riochet D, Labastire L, Auneau JC, Legeard E, Van Tricht M, et al Identification of potential organ donors of advanced age in EDs. Am J Emerg Med. 2012;30(1):170-3. 
8. Traino HM, Alolod GP, Shafer T, Siminoff LA. Interim results of a national test of the rapid assessment of hospital procurement barriers in donation (RAPiD). Am J Transplant. 2012;12(11):3094-103.

9. Witjes M, Kotsopoulos AMM, Otterspoor L, Herold IHF, Simons KS, Woittiez K, et al. The implementation of a multidisciplinary approach for potential organ donors in the emergency department. Transplantation. 2019. https:// doi.org/10.1097/TP.0000000000002701.

10. Prospero. International prospective register of systematic reviews. http//muw.crd.york. ac.uk/PROSPERO/display_record.php?ID=CRD42018068185. Accessed 14 Aug 2018.

11. Higgins JPT, Green S (editors). Cochrane Handbook for Systematic Reviews of Interventions Version 5.1.0 [updated March 2011]. The Cochrane Collaboration, 2011. Available from www.handbook.cochrane.org.

12. Adanir T, Erdogan I, Hunerli G, Unveren G, Dasci H, Cetin HY, et al. The effect of psychological support for the relatives of intensive care unit patients on cadaveric organ donation rate. Transplant Proc. 2014:46(10):3249-52.

13. Beasley CL, Capossela CL, Brigham LE, Gunderson S, Weber P, Gortmaker SL. The impact of a comprehensive, hospital-focused intervention to increase organ donation. J Transpl Coord. 1997;7(1):6-13.

14. Beigee FS, Mohsenzadeh M, Shahryari S, Mojtabaee M. Role of more active identification of brain-dead cases in increasing organ donation. Exp Clin Transplant. 2017;15:60-2.

15. Bires MH. Comparison of consent rates between hospital-based designated requestors and organ procurement coordinators. J Transpl Coord. 1999;9(3): 177-80.

16. Bleakley G. Implementing minimum notification criteria for organ donation in an acute hospital's critical care units. Nurs Crit Care. 2010;15(4):185-91.

17. Feest TG, Riad HN, Collins CH, Golby MGS, Nicholls AJ, Hamad SN. Protocol for increasing organ donation after cerebrovascular deaths in a district general hospital. Lancet. 1990;335(8698):1133-5.

18. Garside J, Garside M, Fletcher S, Finlayson B. Utilisation of an embedded specialist nurse and collaborative care pathway increases potential organ donor referrals in the emergency department. Emerg Med J. 2012;29(3):228-32.

19. Henderson SO, Chao JL, Green D, Leinen R, Mallon WK. Organ procurement in an urban level I emergency department. Ann Emerg Med. 1998;31(4): 466-70

20. Ismail SY, Kums E, Mahmood SK, Hoitsma AJ, Jansen NE. Increasing consent and assent rate for organ and tissue donation: communication about donation-telephone advice by psychologist. Transplant Proc. 2018;50(10): 3017-24.

21. Jansen NE, Van Leiden HA, Haase-Kromwijk BJJM, Van Der Meer NJM, Kruijff EV, Van Der Lely $\mathrm{N}$, et al. Appointing 'trained donation practitioners' results in a higher family consent rate in the Netherlands: a multicenter study. Transpl Int. 2011;24(12):1189-97.

22. Gyllstrom Krekula L, Malenicka S, Nydahl A, Tibell A. From hesitation to appreciation: the transformation of a single, local donation-nurse project into an established organ-donation service. Clin Transpl. 2015; 29(3):185-96.

23. Lenzi JA, Sarlo R, Assis A, Ponte M, Paura P, Araujo C, et al. Family informed consent to organ donation-who performs better: organ procurement organizations, in-hospital coordinators, or intensive care unit professionals? Transplant Proc. 2014:46(6):1672-3.

24. Linyear AS, Tartaglia A. Family communication coordination: a program to increase organ donation. J Transpl Coord. 1999;9(3):165-74

25. Manyalich M, Guasch X, Paez G, Valero R, Istrate M. ETPOD (European Training Program on Organ Donation): a successful training program to improve organ donation. Transpl Int. 2013;26(4):373-84.

26. Mulvania P, Mehakovic E, Wise C, Cass Y, Daly TA, Nathan HM. Successful international collaboration improves family donation conversations resulting in increased organ donation. Transplant Proc. 2014;46(6):2058-65.

27. Sandiumenge A, Ramirez-Estrada S, Mazo C, Rubiera M, Boned S, Dominguez-Gil B, et al. Donor referral from outside the intensive care unit: A multidisciplinary cooperation model using communication apps and redefining refereal criteria. Med Intensiva. 2018. https://doi.org/10.1016/j. medin.2018.08.009. [Epub ahead of print].

28. Siminoff LA, Marshall HM, Dumenci L, Bowen G, Swaminathan A, Gordon N. Communicating effectively about donation: an educational intervention to increase consent to donation. Prog Transplant. 2009;19(1):35-43.

29. Siminoff LA, Traino HM, Genderson MW. Communicating Effectively about Organ Donation: A Randomized Trial of a Behavioral Communication
Intervention to Improve Discussions about Donation. Transplant Direct. 2015;1(2):e5.

30. Stark J, Wikoren B, Martone L. Partners in organ donation: piloting a successful nurse requestor program. Crit Care Nurs Clin North Am. 1994;6(3): $591-8$.

31. von Pohle WR. Obtaining organ donation: who should ask? Organ procurement organization representative. Heart Lung. 1996;25(4): 304-9.

32. Young D, Danbury C, Barber V, Collett D, Jenkins B, Morgan K, et al. Effect of "collaborative requesting" on consent rate for organ donation: randomised controlled trial (ACRE trial). BMJ (Online). 2009;339(7726):899-901.

33. Zier JL, Spaulding AB, Finch M, Verschaetse T, Tarrago R. Improved Time to Notification of Impending Brain Death and Increased Organ Donation using an Electronic Clinical Decision Support System. Am J Transplant. 2017;17(8): 2186-91.

34. Jansen NE, de Groot YJ, van Leiden HA, Haase-Kromwijk BJ, Kompanje EJ, Hoitsma AJ. Imprecise definitions of starting points in retrospectively reviewing potential organ donors causes confusion: call for a reproducible method like 'imminent brain death'. Transpl Int. 2012;25(8):830-7.

35. Kompanje EJ, Jansen NE, de Groot YJ. 'In plain language': uniform criteria for organ donor recognition. Intensive Care Med. 2013;39(8):1492-4.

36. Dominguez-Gil B, Coll E, Elizalde J, Herrero JE, Pont T, Quindos B, et al. Expanding the Donor Pool Through Intensive Care to Facilitate Organ Donation: Results of A Spanish Multicenter Study. Transplantation. 2017; 101(8):e265-72.

37. Caballero F, Puig M, Leal J, Trejo O, Diaz I, Herrera S, et al. A helpful approach to organ donation: from end-of-life care to effective organ transplantation. Am J Transplant. 2018;18(2):528-9.

38. Martinez-Soba F, Perez-Villares JM, Martinez-Camarero L, Lara R, Monzon JL, Fernandez-Carmona A, et al. Intensive care to facilitate organ donation: a report on the experience of 2 Spanish centers with a common protocol. Transplantation. 2019:103(3):558-64.

39. Steering Group of College of emergency medicine and the British Transplantation Society. The role of emergency medicine in organ donation https://bts.org.uk/wp-content/uploads/2016/09/Role-of-emergencyMedicine-in-Organ-Donation.pdf. Accessed June 25, 2018.

40. Chandler JA, Connors M, Holland G, Shemie SD. "Effective" requesting: a scoping review of the literature on asking families to consent to organ and tissue donation. Transplantation. 2017;101(5S Suppl 1):S1-S16.

41. Simpkin AL, Robertson LC, Barber VS, Young JD. Modifiable factors influencing relatives' decision to offer organ donation: systematic review. BMJ. 2009;338:b991.

42. Siminoff LA, Agyemang AA, Traino HM. Consent to organ donation: a review. Prog Transplant. 2013;23(1):99-104.

43. Vincent A, Logan L. Consent for organ donation. Br J Anaesth. 2012; 108(Suppl 1):i80-7.

44. Hulme W, Allen J, Manara AR, Murphy PG, Gardiner D, Poppitt E. Factors influencing the family consent rate for organ donation in the UK. Anaesthesia. 2016;71(9):1053-63.

45. de Groot J, Vernooij-Dassen M, Hoedemaekers C, Hoitsma A, Smeets W, van Leeuwen $\mathrm{E}$. Decision making by relatives about brain death organ donation: an integrative review. Transplantation. 2012;93(12):1196-211.

46. Ebadat A, Brown CV, Ali S, Guitierrez T, Elliot E, Dworaczyk S, et al. Improving organ donation rates by modifying the family approach process. J Trauma Acute Care Surg. 2014:76(6):1473-5.

47. Martinez JM, Lopez JS, Martin A, Martin MJ, Scandroglio B, Martin JM. Organ donation and family decision-making within the Spanish donation system. Soc Sci Med. 2001;53(4):405-21.

48. Witjes M, Kruijff PEV, Haase-Kromwijk B, van der Hoeven JG, Jansen NE, Abdo WF. Physician experiences with communicating organ donation with the relatives: a Dutch nationwide evaluation on factors that influence consent rates. Neurocrit Care. 2019. https://doi.org/10.1007/s12028-01900678-8.

49. Douville F, Godin G, Vezina-Im LA. Organ and tissue donation in clinical settings: a systematic review of the impact of interventions aimed at health professionals. Transplant Res. 2014;3(1):8.

50. Matesanz R, Dominguez-Gil B, Coll E, Mahillo B, Marazuela R. How Spain Reached 40 Deceased Organ Donors per Million Population. Am J. Transplant. 2017;17(6):1447-54.

51. Balleste C, Arredondo E, Gomez MP, Fernandez A, Wolf M, Gunderson S, et al. Successful example of how to implement and develop a deceased organ 
donation system in the Caribbean region: five-year experience of the SEUSA program in Trinidad and Tobago. Transplant Proc. 2015;47(8):2328-31.

52. NHS Blood and Transplant. Organ Donation and the Emergency

Department - A strategy for implementation of best practice. https://

nhsbtdbe.blob.core.windows.net/umbraco-assets-corp/2098/ed-strategy-

final-nov-2016.pdf. Accessed Oct 122018.

\section{Publisher's Note}

Springer Nature remains neutral with regard to jurisdictional claims in published maps and institutional affiliations.

Ready to submit your research? Choose BMC and benefit from:

- fast, convenient online submission

- thorough peer review by experienced researchers in your field

- rapid publication on acceptance

- support for research data, including large and complex data types

- gold Open Access which fosters wider collaboration and increased citations

- maximum visibility for your research: over $100 \mathrm{M}$ website views per year

At $\mathrm{BMC}$, research is always in progress.

Learn more biomedcentral.com/submissions 